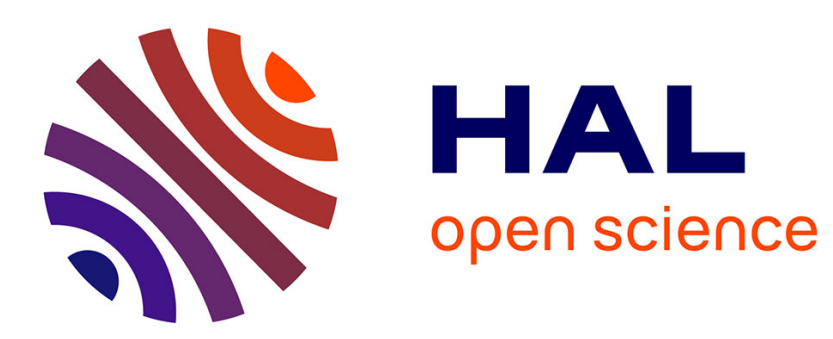

\title{
La guérison est-elle une maladie chronique ? Dette(s), stigmates et conversion \\ Hélène Lecompte
}

\section{To cite this version:}

Hélène Lecompte. La guérison est-elle une maladie chronique ? Dette(s), stigmates et conversion. Revue d'oncologie hématologie pédiatrique, 2014, 2 (4), pp.173-179. 10.1016/j.oncohp.2014.09.005 . halshs-01712598

\section{HAL Id: halshs-01712598 \\ https://shs.hal.science/halshs-01712598}

Submitted on 4 Apr 2018

HAL is a multi-disciplinary open access archive for the deposit and dissemination of scientific research documents, whether they are published or not. The documents may come from teaching and research institutions in France or abroad, or from public or private research centers.
L'archive ouverte pluridisciplinaire HAL, est destinée au dépôt et à la diffusion de documents scientifiques de niveau recherche, publiés ou non, émanant des établissements d'enseignement et de recherche français ou étrangers, des laboratoires publics ou privés. 
Disponible en ligne sur

ScienceDirect

www.sciencedirect.com
Elsevier Masson France

EM|consulte

www.em-consulte.com

ARTICLE ORIGINAL

\section{La guérison est-elle une maladie chronique? Dette(s), stigmates et conversion}

\section{Healing: A chronic condition? Legacy, stigma and conversion}

\section{H. Lecompte}

Centre nantais de sociologie, EA 3260, université de Nantes, chemin de la censive du tertre, BP 81227, 44312 Nantes cedex 3, France

Reçu le 24 février 2014 ; accepté le 29 septembre 2014 Disponible sur Internet le 25 octobre 2014

Résumé Si l'expérience du cancer constitue une épreuve pour les enfants, celle de la guérison les expose également à certaines difficultés. Nombreux sont les travaux médicaux qui analysent les risques (séquelles cardiaques, gonadiques etc.) encourus par les anciens malades atteints de cancer et les études psychologiques alertent sur les difficultés psychiques rencontrées, mais la littérature sociologique sur la guérison, notamment des enfants, est quasiment inexistante. Or, guérir d'un cancer signifie rarement être «bien portant» et le passage du statut d'enfant malade à celui de guéri nécessite certains ajustements, notamment corporels et sociaux. Il s'agit d'expliciter comment une partie de ces ajustements est déterminée par une dette laissée par la guérison, c'est-à-dire non seulement d'étudier le lien entre les deux mais surtout de comprendre les effets de la dette sur les trajectoires sociales des individus. De quelle manière celle-ci peut-elle orienter certaines trajectoires professionnelles? Peut-on parler d'une seule dette et par rapport à qui ? Comment les anciens enfants malades peuvent-ils s'en acquitter et à quel prix? Ces questions ouvrent une réflexion sur la définition même de la guérison dans le cadre du cancer. Il semble que l'état de «guéri» ne corresponde pas toujours à celui de non-malade, ni d'un point de vue physiologique, ni d'un point de vue social et sociétal.

(c) 2014 Elsevier Masson SAS. Tous droits réservés. 


\section{KEYWORDS \\ Child; \\ Cancer; \\ Healing; \\ Legacy; \\ Stigma}

Summary While children suffering from cancer undergo an ordeal, the healing process also puts them through a series of difficulties. Medical research analyzes risks (cardiac and gonadal after effects, for example) incurred by former patients suffering from cancer; psychology studies warn about the psychological difficulties that ensue, but sociology hardly tackles the issue it raises, especially when it deals with children. However, being healed from cancer rarely means being healthy and transitioning from the status of being a sick child to that of being healed does require some adjustments, particularly with regards to the body and to society. It is important to explain how some of these adjustments are determined by the legacy of the cure, i.e. not only to study the link between the two but more importantly to understand the effect of the legacy on the individuals' social trajectories. In what way can it influence professional choices? Can we talk about only one legacy and to whom? How can former sick children cope with it and at what cost? These questions open a new field of reflection about the very definition of healing in the context of cancer treatment. It seems the status of being "healed" does not always match that of being healthy, neither psychologically nor socially or sociologically. (c) 2014 Elsevier Masson SAS. All rights reserved.

\section{Introduction}

Les séquelles organiques et physiologiques des traitements anticancéreux sont nombreuses [1-3]. Elles marquent les corps mais font aussi varier les situations sociales des anciens enfants malades en altérant plus ou moins leur trajectoire scolaire, professionnelle et familiale [4]. Georges Canguilhem l'écrit, la guérison ne doit pas être comprise comme un retour «à l'état antérieur à la maladie» [5], mais plutôt comme un nouvel état. Le passé d'enfant atteint de cancer laisse toute sorte de traces, mais les anciens malades évoquent bien souvent un nouveau rapport au monde qui les distingue plus ou moins des enfants «bien portants ». Ils racontent la manière dont l'expérience de la maladie et de l'hôpital les a transformés et ce que guérir leur a coûté. À partir d'une recherche sociologique consacrée aux effets durables de la prise en charge anticancéreuse sur la construction identitaire des anciens enfants malades [4], nous proposons une réflexion sur la dette laissée par la guérison. Les résultats qui sont présentés ici s'appuient sur une partie des matériaux recueillis, soit 37 entretiens approfondis menés avec d'anciens enfants malades ${ }^{1}$, l'observation de consultations de suivi à long terme et d'un service d'oncopédiatrie pendant six mois.

\footnotetext{
${ }^{1}$ Les anciens malades ont été contactés par deux oncopédiatres qui leur ont expliqué la recherche à l'aide d'un courrier que nous avions rédigé. Nous n'avons sollicité que ceux qui avaient donné leur accord pour participer à l'étude. Les anciens malades ont été choisis à partir de plusieurs variables. L'échantillon devait comporter autant de filles que de garçons, être représentatif de diverses pathologies (tumeurs osseuses, tumeurs musculaires, maladies de Hodgkin, tumeurs cérébrales, lymphomes, leucémies) et de différents âges au diagnostic car nous voulions comparer les effets de cette expérience sur les trajectoires sociales des enfants en fonction de leur âge. Les jeunes adultes interviewés avaient tous entre 1 et 18 ans lorsqu'ils ont été atteints d'un premier cancer. Au moment du diagnostic, ils avaient en moyenne 9,6 ans. Quand nous les avons interviewés, ils avaient en moyenne 22,8 ans.
}

Dans un premier temps, il s'agira de caractériser la dette. Comment qualifier celle-ci? Pour qui, pourquoi et par rapport à qui? Pour la définir et l'analyser, nous partirons de deux injonctions paradoxales faites aux anciens enfants malades : passer outre l'expérience du cancer en la considérant comme une parenthèse pour «aller de l'avant», tout en étant sans cesse renvoyés à ce passé d'enfant malade. Il s'agira ensuite de comprendre pourquoi le «don de soi » permet de s'acquitter de la dette. Enfin, nous montrerons que si tous les anciens malades sont affligés de stigmates, ceux qui bénéficient d'une «meilleure» réinsertion sociale sont aussi ceux qui parviennent à «retourner le stigmate». Nous utiliserons la définition du stigmate proposée par Erving Goffman : «un attribut qui jette un discrédit profond, mais il faut bien voir qu'en réalité c'est en termes de relations et non d'attributs qu'il convient de parler » [6], et ce que l'auteur appelle le retournement du stigmate, soit la conversion de ce dernier en compétence, qui lui confère cette fois-ci une valeur positive.

\section{Le prix de la guérison}

Lors de la consultation de suivi à long terme (aussi appelée consultation de «surveillance»), l'oncopédiatre fait un point sur les séquelles, rappelle les risques auxquels les anciens malades seront potentiellement exposés (nouveau cancer, complications cardiaques, infertilité, etc.), tout en s'assurant qu'ils ont «tourné la page» [6]. Cette consultation rappelle aux enfants et aux jeunes adultes qu'ils ont été malades et que le cancer ne finit jamais tout à fait [7], car il doit toujours être surveillé. Mais paradoxalement, les anciens malades peuvent être encouragés à mener une «vie normale», voire à retrouver leur «vie d'avant ${ }^{2}$.

\footnotetext{
2 L'importance à mener la «vie la plus normale possible» pendant et après les traitements est énoncée par certains professionnels de santé, mais elle est avant tout diffusée par les pouvoirs publics au sein des deux premiers Plans cancer, par l'intermédiaire de l'INCa et
} 
Au-delà de ces deux injonctions pour le moins paradoxales, les conseils médicaux relatifs aux pratiques quotidiennes des anciens malades (en termes d'alimentation, d'activités sportives, de dissuasion à la consommation de drogue et plus largement de limitation des comportements à risque) et les questions qui portent sur leurs aspirations futures (professionnelles et parentales, notamment) éclairent la nature poreuse de la frontière entre prévention, protection et paternalisme. La surveillance du cancer implique en effet une vigilance médicale quant aux pratiques individuelles des anciens malades. Si du point de vue médical ces recommandations et ces questions s'inscrivent dans une logique curative ou préventive, elles peuvent également glisser vers une démarche prescriptive ou éducative. Les anciens malades sont amenés à justifier la manière dont ils gèrent leur corps et leur santé, comme s'ils bénéficiaient d'une seconde vie sur laquelle veille l'oncopédiatre pour s'assurer qu'elle est «correctement» préservée.

Un ancien malade devenu moine bénédictin (26 ans, atteint d'une tumeur cérébrale à 3 ans) raconte que l'oncopédiatre qui s'est occupé de lui aurait aimé qu'il ait un enfant pour transmettre la vie à son tour; comme si c'était l'aboutissement logique d'une vie qui se prolonge malgré la maladie; comme si la guérison devenait absurde si la vie du jeune malade n'était pas perpétuée. C'est également ce qui est dit à Pauline (21 ans, atteinte d'une leucémie à 2 ans) qui fume une vingtaine de cigarettes par jour, sachant pertinemment qu'elle multiplie les chances d'un second cancer, outre les risques cardio-vasculaires auxquels elle est exposée. Lors des consultations de suivi, le médecin ne manque pas de lui rappeler qu'elle n'a pas été soignée pour négliger sa santé maintenant, c'est-à-dire qu'elle n'a pas été traitée «pour rien».

D'une certaine manière, en ne laissant aucune chance aux malades de combattre seuls le cancer, la maladie les met fatalement en dette puisqu'ils deviennent dépendants d'une équipe qui dispose des armes chimiques, des instruments, des compétences, du réseau de coopération, du savoir et d'un lieu qui rassemble les conditions pour combattre le cancer. Catherine Mercier aborde l' «idée de dette» [8] portée par les anciens malades mais il serait plus juste de parler de mise en dette car plus qu'une idée ou une représentation, la dette naît d'un rapport, d'une relation. Elle répond au don fait par une équipe médicale et qui se cristallise bien souvent sur la figure d'un médecin, celui d'une vie prolongée ou plutôt d'une autre vie.

par l'organisation même de la prise en charge. Les pouvoirs publics ont ainsi avancé la nécessité de maintenir certains repères dans les murs de l'hôpital afin de favoriser la réinsertion sociale des anciens malades. La présence de l'école dans les services en est un bel exemple. Les enfants ne comprennent pas toujours pourquoi malgré la particularité de leur situation, ils sont fortement encouragés à poursuivre leur scolarité à l'hôpital. Cela crée une contradiction entre l' "anormalité » à laquelle assigne le statut d'enfant atteint de cancer et le maintien de repères propres au monde hors de l'hôpital, dans les services hospitaliers. Pour aller plus loin sur l'injonction à vivre le plus «normalement» possible pendant et après les traitements, se référer à Lecompte $\mathrm{H}$. Une institution «totale»? Enquête ethnographique d'un service d'oncopédiatrie, [thèse]. Université de Nantes; 2013.
Mais l'oncopédiatre n'est pas le seul à rappeler aux anciens malades qu'ils doivent préserver leur corps et leur santé au nom des risques auxquels ils sont exposés. Clément ( 26 ans, atteint d'un lymphome à 14 ans) ne fume jamais devant ses parents pour éviter un rappel des risques qu'il encoure, comme David (30 ans, atteint d'une maladie de Hodgkin à 14 ans), qui préfère éviter une discussion qui devient toujours conflictuelle. Médecins et parents surveillent les pratiques des anciens malades et la manière dont ils préservent leur santé. Ces derniers doivent protéger leur corps au nom de la «chance» qui est la leur d'être en vie; au nom d'un corps qui a déjà été abîmé et fragilisé ; au nom de la reconnaissance dont ils doivent faire preuve. La dette n'est donc pas seulement une idée ou un fantasme et vient rappeler aux anciens malades qu'ils auraient pu mourir.

Ils sont effectivement désignés par le terme survivors dans les pays anglo-saxons et parfois par celui de «survivants» dans la littérature médicale française, terme qui renvoie le plus souvent aux individus qui ont survécu à la guerre ou encore à une épreuve particulièrement difficile, c'est-à-dire à des individus héroïques qui bénéficient d'un bout de vie supplémentaire dont ils auraient pu être privé $^{3}$. Cette manière de définir les anciens malades rejoint bien souvent les représentations qu'ils ont d'eux-mêmes, des enfants qui auraient pu, ou pour certains qui auraient dû mourir, et qui ne comprennent pas bien à quoi ils doivent leur survie hormis à qui, l'oncopédiatre qui s'est occupé d'eux. L'interrogation sur l'issue positive de la prise en charge au regard des autres vies qui n'ont pu être sauvées demeure souvent. Cette question prend la forme d'un «pourquoi moi », comme si la guérison devait être «méritée». Survivre ne va pas forcément de soi. Comme le dit très justement Catherine Mercier, pour la majorité des anciens malades, l'enjeu est de donner du sens à la maladie mais également à la guérison. La quête de sens n'est pas toujours évidente, notamment car les issues de la prise en charge (survie ou décès) ne peuvent être complètement anticipées ni rationalisées. Elles peuvent alors apparaître comme des situations absurdes, comme Valérie ( 25 ans, atteinte d'une maladie de Hodgkin à 17 ans) le raconte.

-Valérie: Il y a un moment où j'ai... J'ai lâché le morceau ouais, j'ai dit ce que je faisais vraiment (se faire vomir) et ce que je ressentais ouais. J'avais pas beaucoup avancé finalement et la dépression euh... Enfin la dépression. Les idées noires ont duré... Ouais ça s’est étalé sur deux ans, deux ans et demi.

-Qu'est-ce que vous entendez par idées noires?

-Bah idées noires c'est de voir. . (Elle réfléchit) C'est de voir qu'il n'y a rien qui m'intéresse, j'ai rien envie de faire, se demander pourquoi on est là... Alors qu'il y a d'autres gamins qui sont partis quoi, qu'étaient plus jeunes que moi.

-Pourquoi vous vous en êtes sortie, c'est cette question que vous vous posez?

-Ouais. (Silence)

\footnotetext{
${ }^{3}$ En 2007, l'INCa engage d'ailleurs une campagne de communication qui présente les anciens malades comme des «héros ordinaires». Se référer à INCa, Communiqué de presse du 8 janvier 2007, [en ligne], consulté le 2 décembre 2011, URL: http: / / www.ecancer.fr/la-presse/2522-2007/1459-?showall=1
} 
-D'accord, j'avais pas compris ça, j'avais compris que la question que vous vous posiez c'était pourquoi je suis tombée malade mais en fait c'est pas ça...

-Bah... Aussi, si, si, pourquoi je suis tombée malade vous avez raison, si pourquoi parce que j'avais une bonne hygiène de vie donc il n'y avait rien qui... Qui laissait penser. Mais après aussi, qu'est-ce que je fais encore là? Qu'est-ce que je fais encore là parce que j'ai percuté quand j'ai vu tout ce qui se passait dans le service, quand j'ai vu tous les... Tous les enfants, je me suis dit pourquoi moi je m'en suis sortie alors qu'il y avait des très jeunes enfants qui ne sont plus là maintenant quoi! C'est... (Silence).

Valérie ne parvient ni à donner du sens à la maladie, ni à la guérison, ce qui produit une sorte d'errance, comme si elle ne savait plus comment se positionner dans le monde hors de l'hôpital où plus rien ne l'intéresse; un monde fondamentalement injuste car elle n'aurait pas dû tomber malade. Le monde de l'hôpital est également dépourvu de sens parce qu'il emporte des enfants trop jeunes pour mourir alors qu'il prolonge la vie d'enfants plus âgés. À pathologie égale et à traitements thérapeutiques égaux, qu'est-ce qui fait qu'un enfant survit et qu'un autre décède? Outre les marqueurs pronostiques de la maladie et ce que les médecins appellent «la réponse» aux traitements, il reste une part d'incertitude, de chance ou de malchance: la rapidité avec laquelle le diagnostic est posé, les effets secondaires plus ou moins importants en fonction des traitements et des enfants participent à la réussite ou à l'échec thérapeutique. Après l'interrogation sur les causes du cancer, vient celle sur les causes de la guérison, comme si cette dernière était tout aussi mystérieuse que la maladie. Deux sortes de dettes apparaissent alors, celle vis-à-vis du médecin perçu comme celui qui a prolongé la vie, et celle face aux enfants qui n'ont pas survécu, qui prend souvent la forme d'un sentiment de culpabilité. Mais il existe également une dette face à la société qui fait sans cesse ressurgir ce passé d'enfant malade lors de certaines démarches courantes, comme l'acquisition d'un bien immobilier, par exemple.

Lorsqu'on a souffert d'un cancer, les prêts bancaires recouvrent des taux d'intérêt plus importants, notamment car les banques exigent que ces clients estimés à risque, contractent une assurance afin de ne pas perdre d'argent en cas de décès. La convention s'Assurer et emprunter avec un risque aggravé de santé (AERAS) en vigueur depuis le 6 janvier 2007 a été présentée comme un progrès car auparavant, rien n'obligeait les banques à trouver une solution pour répondre à la demande de ces clients. Les anciens malades pouvaient difficilement avoir accès à un prêt bancaire. La convention AERAS aurait permis aux anciens malades de pouvoir contracter un prêt immobilier (inférieur à 32000 euros) ou un crédit à la consommation (inférieur à 17000 euros), contraignant les assurances à les garantir, à condition que l'emprunt soit remboursé avant l'âge de 70 ans. Le risque aggravé de santé concerne les personnes qui ont une espérance de vie possiblement restreinte par rapport au reste de la population. Les banques font remplir un questionnaire médical transmis au médecin-conseil qui dresse un rapport et qui estime les risques. Les assurances, qui protègent plus ou moins leurs clients en cas de complications médicales, prennent une surprime variable, mais toujours significative. Les sociétés d'assurance bon marché n'assurent la personne qu'en cas de décès et ne prennent pas systématiquement les risques d'invalidité en charge, comme une perte de mobilité soudaine ou la déclaration d'un nouveau cancer particulièrement invalidant, par exemple. De même, certaines assurances ne garantissent pas toujours les clients en cas de dégradation significative de leur état, notamment en cas d'Invalidités permanentes totales (IPT) et d'Invalidités temporaires totales (ITT). Soulignons enfin que les assurances demandent $50 \%$ de la cotisation totale dès la première année. Si les anciens malades refusent d'être assurés, les banques, faussement contraintes par la loi qui les obligerait à trouver une solution, renvoient la faute sur leurs clients qui rejettent la proposition qui leur a été faite.

Outre les inégalités évidentes entre les anciens malades et les autres face aux prêts bancaires, le fonctionnement même des assurances et des banques signifie aux premiers qu'ils peuvent mourir à tout moment et comme le dit Céline (26 ans, atteinte d'une maladie de Hodgkin à 14 ans), «il y a toujours quelque chose qui nous rappelle qu'on a été malade. On ne peut jamais tourner définitivement la page. Il y a toujours un moment où les démarches se compliquent ». D'un côté, les anciens malades, encouragés par les pouvoirs publics et par les professionnels de santé, aspirent souvent à retrouver une «vie normale» malgré les séquelles de la maladie et des traitements, et de l'autre, les institutions les considèrent comme des individus «anormaux», au bord de la maladie. Cette double assignation produit une dissonance entre la vie «normale» à laquelle il est permis de rêver pendant la prise en charge du cancer et l'accueil qui leur est fait dans les divers espaces sociaux [4] qu'ils traversent après les traitements.

La guérison stigmatise donc, et implique différentes dettes, la première vis-à-vis du médecin à qui l'on estime devoir sa survie, la seconde face aux enfants qui n'ont pas survécu et la dernière face à la société : il convient de se réinsérer socialement en montrant que l'on correspond aux attentes d'un individu dit normal tout en acceptant d'être définitivement considéré comme une personne malade ou en sursis. Les deux premières dettes amorcent souvent ce qui est verbalisé comme un «besoin d'aider les autres», avancé comme une nécessité à se sentir utile, comme s'il fallait rendre quelque chose pour optimiser cette seconde vie offerte par la guérison. C'est le principe de «don contre don » [9] ${ }^{4}$ théorisé par Marcel Mauss, soit l'acceptation de recevoir, puis l'obligation à rendre (contre don) ce que l'on a reçu (don) selon des règles sociales préétablies afin de rééquilibrer une relation bancale et inégalitaire. Beaucoup d'anciens malades cherchent à s'acquitter de leur dette et y parviennent par divers moyens.

\section{Le contre don comme réparation: «faire sa place»}

S'engager dans la Marine nationale pour «aider les civils» et non pour faire la guerre, travailler en prison pour «faire du social», s'impliquer dans une association de malades, devenir moine bénédictin pour offrir son destin à Dieu, enseigner auprès d'enfants handicapés ou en difficultés scolaires, ou

\footnotetext{
${ }^{4}$ L'auteur évoque la triple obligation donner-rendre-recevoir.
} 
encore offrir une qualité d'écoute et de soutien aux autres; autant de moyens pour se rendre «utile» et donner «de soi ». Certains anciens malades choisissent de revenir dans l'institution hospitalière, cette fois-ci du côté de ceux qui prennent soin ou qui organisent les soins. Stéphanie (19ans, atteinte d'une tumeur osseuse à 12 ans) étudie dans un IUT de gestion et d'administration des entreprises et elle hésite encore entre une spécialisation dans l'import-export et une spécialisation dans la gestion des administrations de santé. La manière dont elle parle de ce qui motive cette deuxième orientation possible est un mélange de don de soi en retour du don qu'elle a elle-même reçu et d'une capacité à comprendre ce que les personnes malades vivent, comme si son histoire lui conférait une certaine légitimité à exercer une profession au sein de l'institution hospitalière.

-Ce serait plutôt de la gestion d'hôpitaux, des choses comme ça?

-Oui c'est ça enfin je sais pas encore vraiment ce qu'ils proposent mais genre je sais pas cadre de santé ou des choses comme ça.

-Ah oui d'accord donc pas seulement le côté administratif parce que c'est effectivement une grosse partie du travail du cadre mais pas seulement.

-Bah c'est travailler à l'hôpital en fait mais dans le milieu de l'administration quoi.

-Pourquoi la santé?

-Bah même si j'ai eu des mauvais souvenirs dans les hôpitaux etc. c'est quand même un milieu qui m'attire.

-Depuis longtemps?

-Bah non depuis que je suis malade je pense, enfin que j'ai été malade. Par exemple, cet été j'ai travaillé un mois à l'hôpital de L. au guichet, et j'ai vraiment aimé en fait. Enfin, j'aime quand même, même si j'ai pas forcément de bons souvenirs, j'aime quand même l'ambiance de l'hôpital.

$-C$ 'est un endroit familier pour vous?

-Ouais. Enfin je pense que c'est pour ça ouais.

-Qu'est-ce que vous aimez dans l'ambiance, les allers et venues, le blanc, les sons parce que vous parlez très bien de la manière dont ces sons et ces odeurs ont pu vous agresser et en même temps, il y a quelque chose dans cette atmosphère qui visiblement vous attire?

-Bah en fait d'un côté je me dis que c'est quelque chose que je connaissais enfin c'est aussi le contact avec les patients parce que je suis passée par là et d'un côté je voudrais... Enfin si je peux aider ou participer et puis si on compare aussi parce que dans les études il y a aussi l'entreprise et la différence avec l'entreprise c'est que c'est pas du tout les mêmes objectifs. Dans l'entreprise le but c'est de faire du profit ou des choses comme ça que là, ça serait un objectif plus social et c'est aussi ce qui m'intéresse.

-Et infirmière c'est quelque chose qui vous a traversé l'esprit ou pas?

-Oui, médecin aussi ça m'a traversé l'esprit mais je sais pas, ça s'est pas fait. Au niveau de mes études il y avait des matières, c'était pas mon truc. Les maths ça allait, mais c'était surtout la physique chimie et je voulais pas faire un bac $S$.

Ce mélange d'attraction et de répulsion envers l'hôpital produit un discours ambivalent sur l'institution. Stéphanie associe le milieu hospitalier à une sorte de bien-être qu'elle peut difficilement verbaliser aussi explicitement. Comment justifier son attirance pour une institution sensée être associée à un passé douloureux? Pourtant, Stéphanie se sent bien à l'hôpital car elle sait s'y situer, c'est-à-dire qu'elle y a sa place. Mais si elle penche plutôt vers cette orientation professionnelle, c'est aussi parce que l'hôpital a une vocation «sociale». Elle oppose ainsi les entreprises auxquelles elle impute un but lucratif, aux hôpitaux auxquels elle attribue un but généreux et «humain», probablement parce qu'elle a bénéficié d'une prise en charge médicale, psychologique et scolaire dans les murs de l'hôpital, de l'accompagnement de travailleurs sociaux (assistante sociale et éducatrice de jeunes enfants) et qu'elle a vécu plusieurs mois dans un centre de rééducation dont elle parle comme d'un espace de rencontres avec d'autres enfants de son âge qui, comme elle, ne rentraient chez eux que le week-end ${ }^{5}$.

Stéphanie boîte et se déplace avec une béquille. Elle porte un stigmate visible qui tend néanmoins à s'effacer dans le milieu hospitalier. En retournant dans l'institution dans laquelle elle s'est longtemps (re)construite, Stéphanie renoue à la fois avec son passé et exerce un contrôle sur le stigmate. On peut supposer que ce dernier devient un atout à l'hôpital qui lui confère une certaine légitimité auprès des patients, bien que la différence de taille entre ses deux jambes ne dise rien de son passé de malade, seulement qu'elle est handicapée. En revanche, le stigmate renseigne sur la manière dont Stéphanie peut être disqualifiée dans le monde hors de l'hôpital, au sein d'une institution qui s'occupe de personnes malades. Cette situation permet donc à Stéphanie d'être tant du côté de l'institution hospitalière que du côté des patients - groupe auquel elle s'identifie facilement puisqu'elle est «passée par là»-de s'aligner sur le groupe (les individus malades) et hors du groupe (les individus «bien portants»), de s'acquitter de sa dette (contre don) et enfin d'optimiser son passé d'enfant malade en le valorisant dans la sphère professionnelle.

\section{Le retournement du stigmate}

Lors de la $30^{\mathrm{e}}$ journée d'oncologie pédiatrique organisée à l'Institut Gustave Roussy en 2009 [10], le cofondateur de l'association Jeunes solidarité cancer (JSC) se présente comme «un malade de haut niveau». Il s'était déjà présenté ainsi lors d'un entretien d'embauche dans le secteur de l'industrie pharmaceutique car «qui de mieux qu'un ancien malade peut comprendre un patient et parler aux médecins?» Il investit son passé d'enfant malade comme une compétence professionnelle et il ne cherche plus à dissimuler son stigmate car «la divulgation volontaire entre dans l'itinéraire moral dont elle marque l'une des étapes.

\footnotetext{
${ }^{5}$ Mais transposer le fonctionnement des institutions dans lesquelles elle a été traitée aux autres, c'est occulter la singularité des services de pédiatrie, notamment caractérisés par une organisation institutionnelle qui leur est spécifique (présence parentale continue, éducateurs de jeunes enfants, enseignants, clowns, musiciens etc.) et par la triangularité de la relation thérapeutique. Dans les services d'oncologie adultes, il y a des assistants sociaux mais le lien avec le travail est rompu. Le conjoint ne peut dormir à l'hôpital ni bénéficier d'un congé longue durée pour accompagner le malade au quotidien, contrairement aux parents d'enfants malades qui disposent de l'Allocation journalière de présence parentale (AJPP).
} 
Ajoutons que, dans les autobiographies de personnes stigmatisées, c'est typiquement cette étape qui est décrite comme étant la dernière, celle de la maturité et de la complète adaptation, sorte état de grâce » [6].

Mathieu est âgé de 24 ans et a souffert d'une leucémie à 16 ans. Lorsque le diagnostic tombe, il est en CAP boulanger et sa voie professionnelle est toute tracée. Au début de la prise en charge, l'oncopédiatre lui dit qu'il doit changer d'orientation professionnelle à cause d'une future fatigabilité qui l'empêchera de rester debout toute la journée et de se soumettre aux horaires imposés par le métier. Malgré son désir d'être boulanger qui lui vient de loin (depuis qu'il est tout petit), à aucun moment Mathieu n'envisage ce changement d'orientation comme un événement malheureux. Au contraire, il en parle comme d'une opportunité dont il s'est saisi. Cette longue hospitalisation est l'occasion de réfléchir à un nouveau projet professionnel et de repartir «sur de nouvelles bases ». La maladie et la prise en charge hospitalière qu'elle a nécessitée lui auraient permis d'ouvrir ses perspectives professionnelles. Reconnu travailleur handicapé quand il quitte l'hôpital, Mathieu suit différentes formations grâce à la mission locale et prend le temps de s'essayer à différents métiers : palefrenier, facteur, ambulancier et brancardier.

-Ça a été compliqué de faire des formations avec ce statut ou?

-Euh très en fait. Ça effraie... Quand. . c'est pas que ça effraie, mais je sais pas. Parce qu'en fait, j'ai eu un premier entretien à la clinique $Z$ en fait pour... Être brancardier et je sais pas si c'est à cause de ça mais dès que je leur ai parlé que j'ai été malade, ils ont eu du recul, enfin ils ont hésité, à me prendre.

-Est-ce que tu sais pourquoi?

-Non, je sais pas.

-Mais tu as senti une réticence de leur côté, à partir du moment où tu leur as dit ça.

-Voilà... Même quand, parce que je me suis présenté en intérim aussi et donc au départ, on se présente et tout, et une fois qu'ils te proposent du travail pour que tu commences à travailler, ça va très bien mais après en fait, je leur ai dit que j'étais travailleur handicapé, et là ils demandent la cause, et après ils te proposent plus rien en fait, ils t'appellent plus. Ça m'est arrivé plusieurs fois.

-Pourquoi tu leur as dit?

-Bah parce qu'en fait, j'ai ma reconnaissance... enfin je sais pas moi je le dis parce qu'ils posent la question sur le dossier.

-Mais tu ne leur as pas dit au début?

-Non, je leur ai dit qu'après. Parce qu'en fait j'avais déjà eu l'expérience d'y aller, de leur dire dans le dossier, et bah après ils m'appelaient pas, enfin je les appelais mais ils me rappelaient pas. Et en fait, j'ai fait le test pour voir si ça venait de ça, donc j'ai été dans une autre agence, donc eux, ils m'ont proposé du travail, j'ai accepté et une fois que j'ai accepté et que j'ai fait mon contrat d'une semaine ça y est, et après je leur ai dit que j'étais travailleur handicapé et après, il n'y avait plus rien. Ils me proposaient plus rien! Je sais pas ils ont peur que s'ils m'arrivent quelque chose sur le lieu de travail ou je sais pas quoi, que ça leur retombe dessus, enfin je sais pas.

Bien que l'on retrouve certaines tensions évoquées par d'autres anciens malades et notamment l'exclusion que produit le statut handicapé, Mathieu se saisit de ce double statut: ancien malade-handicapé ${ }^{6}$. Sa parfaite maîtrise des règles d'hygiène propres à l'hôpital (intériorisées lorsqu'il était malade) et sa connaissance du fonctionnement global et singulier de l'institution hospitalière sont finalement des atouts qui lui permettent d'être recruté comme animateur dans un service de rééducation pour «distraire les malades», comme il dit. Il est «passé de l'autre côté» et son passé vient légitimer sa place à l'hôpital.

Mathieu est également engagé dans une association de malades qui organise des sorties et des activités pour les enfants atteints de cancer (l'AOPA). Cette association tient une place importante au sein du service dans lequel il a été soigné lorsqu'il était adolescent et certains professionnels de santé y sont également engagés. Son passé est en quelque sorte réactualisé au présent, mais d'une autre manière. Mathieu a construit une passerelle entre le monde de l'hôpital et celui du dehors qui lui permet de circuler aisément et de garder un pied dans chacun d'entre eux. L'état de «guéri » devient un liant qui fait communiquer les deux mondes. Si Mathieu fait partie des anciens malades qui s'en sortent le mieux - au même titre que ceux qui ont été malades tout petits-c'est parce qu'il n'est ni dans la minimisation de ce qui lui est arrivé, ni dans l'illusion de pouvoir retrouver une «vie normale». Il le dit d'ailleurs explicitement: «c'est ma vie à moi, elle est différente. Je le sais». Contrairement à la plupart des enfants qui s'apprête à quitter l'hôpital, Mathieu n'espère pas retrouver sa «vie d'avant» parce qu'il sait depuis le début des traitements que celle-ci est définitivement perdue. Son passé d'enfant malade lui offre une autre vie et comme AnneChantal Hardy le souligne, «si les situations objectives sont assez directement corrélées aux pathologies, les situations subjectives suivent des logiques différentes et montrent que ces «bonnes» insertions sociales et professionnelles ne se sont pas déroulées «malgré », mais «avec » la maladie » [11] 7 .

\section{Conclusion}

Si les anciens malades s'estiment souvent «chanceux» de pouvoir bénéficier d'une seconde vie, ils constatent également, tout comme leurs parents, que la guérison a un coût physiologique, organique, social, psychique [12], symbolique et financier. Malgré ce prix déjà élevé, la plupart des anciens malades s'estiment encore redevable et cherche

\footnotetext{
${ }^{6}$ Il ne sait d'ailleurs pas bien s'il doit ses difficultés à être embauché à son passé de malade ou à son statut handicapé, c'est-à-dire ce qui le stigmatise davantage dans la sphère professionnelle. Le statut handicapé constitue à la fois une reconnaissance et un stigmate, comme pour beaucoup d'anciens malades.

7 Nous excluons ici les anciens malades qui ont souffert d'une tumeur cérébrale car la plupart d'entre eux cumule les séquelles, notamment cognitives, et celles-ci entravent sévèrement les trajectoires scolaires puis professionnelles des jeunes adultes. Néanmoins, notre recherche montre que ce ne sont pas toujours les individus qui souffrent des séquelles les plus visibles et particulièrement lourdes d'un point de vue clinique qui «s'en sortent» le «moins bien». Par exemple, la fatigue chronique, qui constitue pourtant une séquelle invisible, empêche grandement la fluidité des parcours.
} 
à s'acquitter de la dette la plus importante laissée par la guérison, «mériter» cette seconde vie. Nombreuses sont les manières de se défaire petit à petit de ce lourd tribut. L'exercice d'une profession à l'hôpital constitue l'un des moyens (et sans doute le plus explicite) pour compenser cette posture débitrice.

Mais le retour dans le milieu hospitalier permet également de transformer l'expérience de la maladie et de l'hôpital en compétences. Il s'agit alors moins de s'acquitter d'une dette par l'intermédiaire d'un contre don que de s'aménager une plus grande marge de liberté en reprenant le dessus sur ce qui constitue initialement un handicap, une entrave et c'est bien là l'intérêt de l'inversion du stigmate. Si le cancer est guéri, les personnes ne le sont pas forcément et celles qui se réinsèrent «le mieux» socialement sont justement celles qui peuvent valoriser leur différence, comme si, au lieu de traîner ce passé comme un poids mort, la possibilité de le faire reconnaître dans différents espaces sociaux (professionnel, associatif et amical essentiellement) compensait certaines pertes. Difficile alors d'avancer qu'il existe une «sortie» du cancer [7], tant le statut d'enfant "guéri » est ambigu. Il nécessite à la fois une identification au statut d'enfant «normal» (retrouver sa vie «d'avant» et considérer la prise en charge comme une parenthèse) et au statut d'enfant «anormal » (consultations de suivi à long terme, rappels à l'ordre, séquelles, risques de cancer secondaire, difficulté d'accès au prêt bancaire etc.).

Qu'elles soient visibles ou non, les traces de ce passé avec le cancer interrogent la guérison, associée la plupart du temps à un état de non-malade, c'est-à-dire «normal». Dans un contexte sociétal où l'enfant «est passé au peigne fin d'une normalité qui se fait toujours plus pressante et exigeante» [13], notamment à l'école, il est primordial d'engager une réflexion sur le statut des anciens enfants atteints de cancer, à mi-chemin entre celui de malade, d'handicapé et de «guéri». Notre recherche a étudié la manière dont cette course à la «normalité » est encouragée par les pouvoirs publics, par certains professionnels de santé et par certaines institutions comme l'école ${ }^{8}$ qui souhaitent que les enfants reprennent leur vie là où ils l'avaient laissée, comme si l'expérience du cancer et de l'hôpital pouvait constituer une parenthèse. Il est sans doute nécessaire de rompre avec l'illusion d'un retour à une «vie normale » après la fin des traitements et plus largement avec cette obsession à la norme, à laquelle la plupart des anciens enfants malades ne pourra, de toute façon, jamais totalement correspondre. Si l'état de «guéri » reste encore associé à celui de «bien portant» dans la plupart des espaces sociaux, ce n'est qu'au prix de ce pas de côté que les représentations sociales évolueront.

\footnotetext{
${ }^{8}$ Lorsque les anciens malades reviennent à l'école, les enseignants considèrent souvent qu'il faut les traiter comme les autres, notamment si les séquelles sont invisibles. La fatigue chronique est très mal connue et reconnue et certains anciens malades sont accusés de «tirer au flanc».
}

\section{Déclaration d'intérêts}

L'auteur déclare ne pas avoir de conflits d'intérêts en relation avec cet article.

\section{Remerciements}

Je voudrais d'abord remercier les jeunes adultes de m'avoir livré leur histoire. Je souhaite également exprimer ma reconnaissance aux enfants, à leurs parents et aux différents acteurs de prise en charge en oncologie pédiatrique qui m'ont accueilli dans leur service pendant six mois. En second lieu, je souhaite remercier la Fondation de France d'avoir financé ma thèse de doctorat. Enfin, je voudrais exprimer ma gratitude à Franck Bourdeaut, Anne-Chantal Hardy et Cédric Le Bodic pour leurs conseils et leur relecture.

\section{Références}

[1] Chaume AG, Nerger C, Cathébras P. Séquelles et qualité de vie chez de jeunes adultes survivants de cancers pédiatriques. Rev Med Interne 2007;28:450-7.

[2] Langeveld NE, Stam H, Grootenhuis MA, Last BF. Quality of life in Young adult survivors of childhood cancer. Support Care Cancer 2002;10:579-600.

[3] Lackner $H$, Benesh $M$, Schagerl S, Kerbl R, Schwinger $W$, Urban C. Prospective evaluation of late effects after childhood cancer therapy with a follow-up over 9 years. Eur J Pediatr 2000;159(10):750-8.

[4] Lecompte H. Une institution «totale»? Enquête ethnographique d'un service d'oncopédiatrie [thèse]. Université de Nantes; 2013. p. 275-91.

[5] Canguilhem G. Le normal et le pathologique. Paris: PUF; 1972. p. 130.

[6] Goffman E. Stigmate. Les usages sociaux du handicap. Paris: Les éditions de Minuit; 1975. p. 13.

[7] Hardy A-C, Lecompte H. Lorsque l'enfant guérit. . Étude sociologique des effets à long terme de la prise en charge du cancer chez les enfants et leurs parents, rapport de recherche pour la Ligue nationale contre le cancer, Nantes; 2009.

[8] Mercier C. L'idée de guérison en oncologie pédiatrique. À partir de 8 observations. Revue critique de la littérature [thèse]. Université de Nantes; 1997. p. 111.

[9] Mauss M. Essai sur le don. Forme et raison de l'échange dans les sociétés archaïques. In: Sociologie et anthropologie. Paris: PUF; 1968. p. 145-273 [L'auteur explique la triple obligation donner-rendre-recevoir].

[10] Jeunes solidarité cancer. «Le point de vue des patients», $30^{\mathrm{e}}$ journée d'oncologie pédiatrique: devenir adulte après traitement d'un cancer dans l'enfance, Villejuif ; 26 janvier 2009.

[11] Hardy A-C. Travailler à guérir. Sociologie de l'objet du travail médical. Rennes: Presses de l'EHESP; 2013. p. 236.

[12] Brun D. L'enfant donné pour mort. Enjeux psychiques de la guérison. Paris: Dunod; 1989.

[13] Gavarini L. La passion de l'enfant. Filiation procréation et éducation à l'aube du XXI siècle. Paris: Éditions Denoël; 2001. p. 337. 